\title{
Characteristics of Rhizosphere Fungal Community in Spring Wheat Under Different Rotation Fallow modes
}

\author{
Gongfu Shi ${ }^{1,2}$, Jing Fang ${ }^{1,2}$, Shuli Wei ${ }^{1,2}$, Yuchen Cheng ${ }^{2}$, Xiangqian Zhang ${ }^{2}$, Yu An ${ }^{1,2}$, Zhanyuan Lu ${ }^{1,2, *}$, and Xiaoqing \\ Zhao $^{1,2, *}$ \\ ${ }^{1}$ School of Life Science, Inner Mongolia University; Key Laboratory of Herbage \& Endemic Crop Biotechnology, Ministry of Education, \\ China \\ ${ }^{2}$ Inner Mongolia Academy of Agricultural \& Animal Husbandry Sciences/ Inner Mongolia Conservation Tillage Engineering Technology \\ Research Center/ Inner Mongolia Key Laboratory of Degradation Farmland Ecological Restoration and Pollution Control, China
}

\begin{abstract}
Rhizosphere fungi play an important role in agricultural ecosystems. Their activities and interactions greatly affect various ecosystem processes related to nutrient cycling, soil borne diseases and crop health. The aim of this study was to investigate the effects of rotation fallow mode of spring wheat, potato, fallow and spring wheat, rape, fallow on rhizosphere fungi community structure and functional fungi composition of spring wheat. In this paper, high-throughput sequencing and bioinformatics were used to systematically analyze the diversity, composition and abundance of functional fungi in the rhizosphere of spring wheat at flowering stage. The results showed that: compared with WWW, rotation fallow mode could increase the diversity of rhizosphere fungi and eliminate a small number of specific fungi; The dominant phylum of spring wheat rhizosphere fungi were Ascomycota (32.72-47.99\%), Basidiomycota (8.39-34.21\%) and Mortierellomycota (2.05-18.42\%). Rotation fallow mode significantly increased the relative abundance of Mortierellomycota and Chytridiomycota; The relative abundance of Basidiomycota decreased significantly $(\mathrm{P}<0.05)$; By increasing the relative abundance of potentially beneficial genera of Mortierella, unidentified_Mortierellales_sp and Atractiella, Reducing the relative abundance of potentially harmful fungi genera of Fusarium, Plectosphaerella and Alternaria to a certain extent kept the soil healthy. Compared with other modes, WFP is most likely to cause rhizosphere fungi community structure to develop in favor of plant health.
\end{abstract}

\section{Introduction}

Soil is the basis of crop growth, it can provide all kinds of nutrients for crop growth. The quality of soil determines the yield and quality of crops. With the accelerated process of globalization, human activities and other human factors aggravate the frequent occurrence of extreme weather such as drought, waterlogging and acid rain, which further highlights the problem of soil quality [1]. Rhizosphere microorganisms play an important role in agricultural ecosystems, and their activities and interactions greatly affect various ecosystem processes related to nutrient cycling, soil borne diseases and soil productivity [2]. Rhizosphere microorganisms have the closest relationship with crops, which can selectively "domesticate" specific beneficial microorganisms, promote the transformation of plant nutrients, and facilitate their better growth and development [3]. Rhizosphere soil fungi are an important part of rhizosphere microorganisms. Fungal community diversity and community composition can cause changes in soil quality and affect the growth and development of crops [4].

Soil fungi can be divided into harmful and beneficial according to their functions in farmland ecosystem. Some soil fungi can infect crops and cause plant diseases; Some soil fungi can inhibit the infection of pathogenic fungi to plants, promote plant growth or decompose plant residues [5-6]. Research shows, Different soil cultivation crops and environmental conditions can cause changes in soil fungal community structure [7-8]. Soybean continuous cropping increased the number of Fusarium, the infection rate of root rot increased significantly [9]. Long term continuous cropping of potato resulted in a decrease in the number of rhizosphere fungal, but an increase in the number of fungi, The number of Phoma, Myrothecium and Verticillium in wheat potato rotation and fallow soil decreased significantly, and crop yield and water use efficiency increased significantly [8]. Therefore, rotation fallow can improve soil microenvironment, optimize rhizosphere soil fungal community structure, reduce the occurrence of soil borne

*Corresponding author: Izhy2811@163.com

zhaoxq204@163.com 
diseases and increase crop yield by adjusting cultivation methods and crop species.

As mentioned above, most of these studies focus on the continuous cropping, rotation or long-term fallow the impact on the community structure of soil fungi, and adding leisure farming for crop rotation system of related research is less, especially the use of the same crops rhizosphere fungal diversity and function to reflect the rotation fallow implementation cycle effect research rarely reported. In this study, high-throughput sequencing and bioinformatics were used to systematically analyze the variation trend of rhizosphere fungal diversity of spring wheat under different rotation fallow modes, and to clarify the variation rule of rhizosphere fungal composition and beneficial fungal abundance of spring wheat under different rotation fallow modes. In order to improve the soil quality, screen out the best rotation fallow mode and maintain the healthy and sustainable development of soil ecological environment to provide scientific and effective theoretical basis.

\section{Materials and methods}

\subsection{Experimental site description}

The experimental site is located in the scientific observation and experimental station of soil management and ecological restoration of Tenihe, Inner Mongolia Academy of agriculture and animal husbandry Sciences

(E12048', N49 55', $650 \mathrm{~m}$ above sea level) , This area is located in the middle temperate semi-arid continental grassland climate zone, with an annual average temperature of $2.2{ }^{\circ} \mathrm{C}$, The frost free period is 90-105 days, and the annual average precipitation is 373$474 \mathrm{~mm}$. The precipitation from June to September accounts for about $80 \%$ of the annual precipitation, The effective rainfall in the whole growth period of spring wheat in 2019 is $223.9 \mathrm{~mm}$ 。 The soil type is chernozem, with $110-120 \mathrm{mg} / \mathrm{kg}$ of available $\mathrm{N}, 144 \mathrm{mg} / \mathrm{kg}$ of available $\mathrm{K}$ and $20 \mathrm{mg} / \mathrm{kg}$ of available $\mathrm{P}$, and $\mathrm{pH}$ value of 6.8 .

\subsection{Experimental design}

The long-term treatment was started in 2016, There are five modes. Spring wheat ${ }^{2016}$ - Spring wheat ${ }^{2017}$-Spring wheat $^{2018}$ (WWW); Spring wheat ${ }^{2016}$-Rape ${ }^{2017}$-Fallow ${ }^{2018}$ (WRF); Spring wheat ${ }^{2016}$-Potato ${ }^{2017}$-Fallow 2018 (WPF); Spring wheat ${ }^{2016}$-Fallow ${ }^{2017}-$ Rape $^{2018}$ (WFR); Spring wheat $^{2016}$-Fallow ${ }^{2017}$-Potato ${ }^{2018}$ (WFP). The split zone design was adopted in the experiment. Each process was repeated three times, and there were 15 cells in total. The cell area was $9 \mathrm{~m}^{2}$, the space between groups was $0.5 \mathrm{~m}$, and $1 \mathrm{~m}$ bandwidth protection row was set. Spring wheat was sown on May 5, 2019, with a planting amount of $300 \mathrm{~kg} / \mathrm{hm}^{2}$, spacing of $0.15 \mathrm{~m}$ between rows. Urea was applied to the planting: $60 \mathrm{~kg} / \mathrm{hm}^{2}$, diammonium phosphate: $180 \mathrm{~kg} / \mathrm{hm}^{2}$, potassium sulfate: $30 \mathrm{~kg} / \mathrm{hm}^{2}$, no fertilizer was applied in the later stage, and other management methods were the same as those in the field.

\subsection{Sampling of rhizosphere soil}

At the flowering stage of spring wheat, 15 spring wheat with uniform growth were selected in the plot, and the wheat was excavated with soil with a sampling shovel about $10 \mathrm{~cm}$ around the root and $20 \mathrm{~cm}$ deep, After removing the large soil, the rhizosphere soil was taken with sterile brush, and the $0.5 \mathrm{~mm}$ sterilized stainless steel mesh screen was used to screen out the impurities. The impurities were separated into five $5 \mathrm{ml}$ sterilized centrifuge tubes, marked, frozen in liquid nitrogen, and stored in the refrigerator at $-80^{\circ} \mathrm{C}$.

\subsection{Extraction of total DNA from soil}

CTAB method was used to extract total DNA from soil samples. The concentration and purity of DNA were detected by nanodrop2000uv-Vis, and the quality of DNA was detected by $1 \%$ agarose gel electrophoresis. Take an appropriate amount of sample into the centrifuge tube and dilute the sample to 1 ng with sterile water/ $\mu 1$. Store at $-80^{\circ} \mathrm{C}$.

\subsection{DNA sample amplification and construction of Illumina MiSeq sequencing Library}

ITS1 gene of fungi was selected for sequencing, The primers were ITS1F ( 5'-CTTGGTCATTTA GAGGAAGTAA-3' ) and ITS2R ( 5'-GCTGCGT TCTTCATCGATGC-3' [11]. Using diluted genomic DNA as template, the specific sequence was amplified by SensoQuest PCR (Lab Cycler 96, Germany). The PCR parameters were pre denatured at $94{ }^{\circ} \mathrm{C}$ for $3 \mathrm{~min}$; 35 cycles $\left(94{ }^{\circ} \mathrm{C}\right.$, denatured for $30 \mathrm{~s}$; Annealed at $55^{\circ} \mathrm{C}$ for $30 \mathrm{~s} ; 72{ }^{\circ} \mathrm{C}$ for $45 \mathrm{~s}$ ); Extend at $72{ }^{\circ} \mathrm{C}$ for $10 \mathrm{~min}$. PCR products were detected by electrophoresis with agarose gel of $2 \%$ concentration. The library was constructed by TruSeq DNA PCR free library preparation kit of Illumina company, After qubit quantification and library detection, the constructed library was sequenced by NovaSeq 6000 [12].

\subsection{Data processing and bioinformatic analysis}

The sequencing sequence information was filtered, spliced and quality controlled by qime (v1.8.0) software [13-14], and then OTU (operational taxonomic units) clustering annotation was performed. OTUs are classified and counted at $97 \%$ similarity level by RDP classifier Bayes algorithm [15]. The diversity of alpha was analyzed by using mothur software (version 1.31.2) [16]; Principal coordinate (PCoA) dimensionality reduction map was used to show the community similarity of different rotation fallow modes. LEfse and other statistical analysis methods were used to test the significance of species composition and community results of different rotation fallow modes. One-way 
analysis of variance (ANOVA) for potentially beneficial and harmful fungi using IBM SPSS Statistics 22.0.

\section{Results}

\subsection{Diversity and richness of rotation fallow treatments}

A total of 2,536,710 valid reads were generated from 30 soil samples. The high quality reads were clustered into 2583 microbial OTUs at $97 \%$ similarity. Dilution curve is a curve describing the diversity of samples in a group, which can directly reflect the rationality of sequencing data amount. With the increase of the number of sequenced samples, the dilution curves of each sample tended to be flat, indicating that almost all rhizosphere fungi were included in the sequenced samples (Fig. 1).

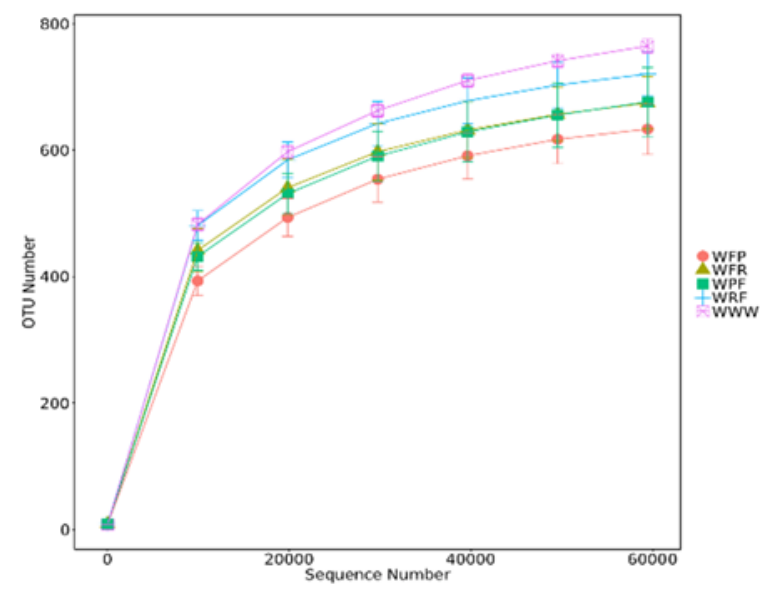

Fig. 1. Sample dilution curve

Alpha diversity can directly reflect the richness and diversity of microbial community in the sample. Fungal community richness (Chaol, Ace) and diversity
(Shannon, Simpson) were significantly different among different cropping rotation fallow mode (Table 1).

Compared with WWW, WRF, WPF and WFR had no significant difference in fungal community richness, but the fungal community diversity was significantly increased $(\mathrm{P}<0.05)$; WFP Shannon and Simpson index were higher than WWW, while Chaol and ACE index were significantly lower than $\mathrm{WWW}(\mathrm{P}<0.05)$. The results showed that, compared with continuous cropping, crop rotation fallow can significantly increase the diversity of fungal communities and decrease specific species. Rotation crops and rotation sequence will affect the rhizosphere fungal community composition to a certain extent.

\subsection{Species composition of rotation fallow treatment}

There were significant differences in fungal community composition among different cropping rotation fallow mode. At the phylum level, Ascomycota (32.72-47.99\%), Basidiomycota (8.39-34.21\%) and Mortierellomycota $(2.05-18.42 \%)$ were the three most abundant phylum in the rhizosphere fungi of spring wheat, and the total relative abundance WWW was significantly higher than that of rotation fallow mode. The types and numbers of fungal phyla with a total relative abundance of more than $60 \%$ are different among the models. WWW has two phyla, Ascomycota and Basidiomycota, and their relative abundances are $41.57 \%$ and $34.02 \%$. There were three phyla of the same species in both WRF WPF and WFR, Ascomycota 、Basidiomycota and Mortierellomycota, respectively. WFP has 3 phyla, Ascomycota, Mortierellomycota and Rozellormycota, the relative abundances are $32.72 \%, \quad 18.42 \%$ and $12.10 \%$ respectively. Compared with WWW, the relative abundance of Mortierellomycota and Chytridiomycete

Table 1. Alpha diversity index of rhizosphere fungi

\begin{tabular}{ccccc}
\hline & Shannon & Simpson & Chao & ACE \\
\hline WWW & $5.74 \pm 0.30 \mathrm{~b}$ & $0.91 \pm 0.02 \mathrm{~b}$ & $766.77 \pm 13.11 \mathrm{a}$ & $755.92 \pm 16.23 \mathrm{a}$ \\
WRF & $6.49 \pm 0.26 \mathrm{a}$ & $0.97 \pm 0.01 \mathrm{a}$ & $722.84 \pm 38.36 \mathrm{ab}$ & $711.76 \pm 43.84 \mathrm{ab}$ \\
WPF & $6.44 \pm 0.08 \mathrm{a}$ & $0.97 \pm 0.00 \mathrm{a}$ & $686.79 \pm 67.59 \mathrm{ab}$ & $669.15 \pm 57.69 \mathrm{ab}$ \\
WFR & $6.43 \pm 0.37 \mathrm{a}$ & $0.96 \pm 0.02 \mathrm{a}$ & $680.59 \pm 42.63 \mathrm{ab}$ & $667.03 \pm 42.56 \mathrm{ab}$ \\
WFP & $5.63 \pm 0.04 \mathrm{~b}$ & $0.95 \pm 0.00 \mathrm{ab}$ & $629.32 \pm 42.34 \mathrm{~b}$ & $621.72 \pm 40.77 \mathrm{~b}$ \\
\hline
\end{tabular}

in rotation fallow modes was significantly increased, while the relative abundance of Basidiomycota was significantly decreased $(\mathrm{P}<0.05)$. The relative abundance of Rozellormycota in WFP was significantly higher than that in the other four modes $(\mathrm{P}<0.05)$ (Fig. 2a). At the genus level, the relative abundance of Coniophora and Chaetomiaecum-Unidentified in WWW increased significantly the relative abundance of Mortierella, Plectosphaerella and Mortierellaceae -Unidentified was significantly decreased. The relative abundance of
Alternaria in WFP was the lowest, significantly lower than that of WWW, WRF and WPF $(\mathrm{P}<0.05)$ (Fig. 2b).

\subsection{Analysis on species factors of group differences}

We used the Unweighted Unifrac algorithm in principal coordinate analysis to measure the differences in fungal community structure among spring wheat rhizosphere 

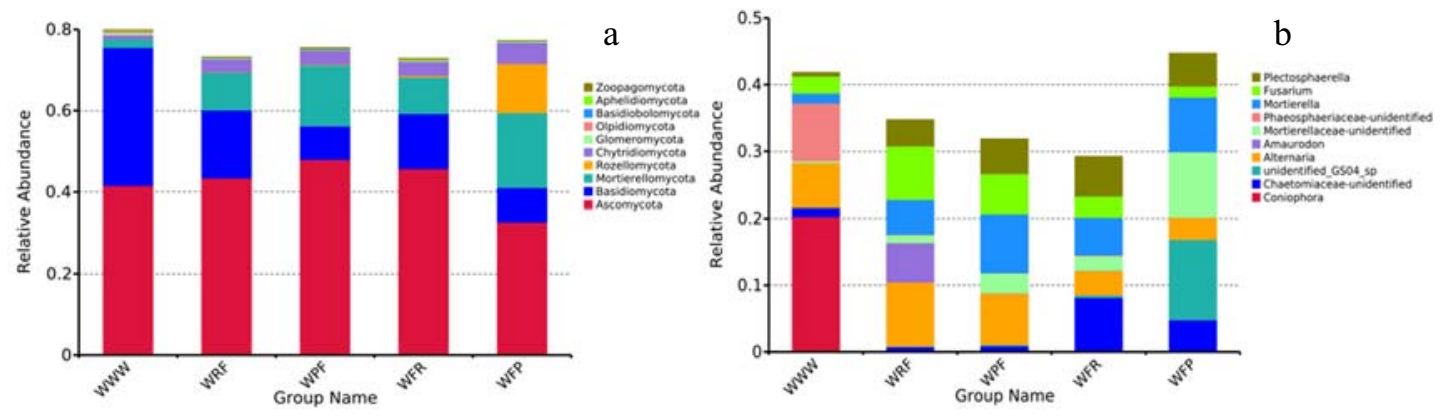

Fig. 2. Fungal community composition under different rotation fallow modes

samples with different rotation fallow modes and analyze the reasons for the differences in fungal community structure. The PCoA results showed that the five model sample points were divided into three main regions, WWW in an independent region, WFP in another independent region, and WRF, WPF and WFR in the same region. In the same position, the dispersion of sample points is small, and the community composition of sample points is relatively similar. The results showed that cropping rotation and fallow was the main factor causing the community differences. Compared with WWW and WFP, the fungal community composition of WRF, WPF and WFR was relatively similar (Fig. 3).

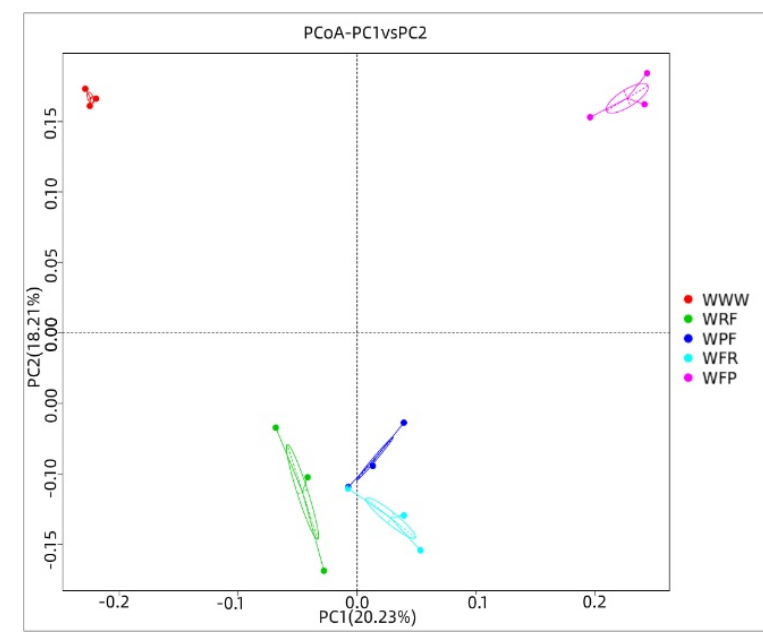

Fig. 3. Fungal community composition under different rotation fallow modes

\subsection{Analysis of species difference among groups}

The LEfSe analysis was used to analyze the fungal composition of soil samples with different cropping rotation fallow modes and the species with significant differences in sample components were identified. When the LDA threshold was 4.0, there were significant differences in the relative abundances of 76 fungal groups from the five modes. At different taxonomic levels, they were distributed in 5 phyla, 8 classes, 14 orders, 15 families, and 18 genera. The number of
WWW, WRF, WRF, WPF and WF differential fungal is $20,10,15,10$ and 21, respectively. At the genera level, the WWW mode enrichment genera are Coniophora, Corticiaceae_sp, Abrocybe and Parastagonospora, of which Coniophora has the largest abundance (LDA value is the largest) and plays the most important role. Fusarium, Amaurodon and Unidentified Mortierellales_sp were the most abundant genera in WRF, and Fusarium played a more important role in WRF. Plectosphaerella, Phallus and Chaetomium were enriched in the WFR mode, and the abundance of Plectosphaerella was the highest and played the largest role. The enrichment in WPF mode belongs to Mortierella, Trichocladium and Cladosporium, in which Mortierella plays a major role. The genera enrichment in the WFP mode were Unidentified GS04 SP, Atractiell and Mortierella, in which Atractiell playe $\overline{-}$ a major role (Fig. 4).

\subsection{Analysis of potentially beneficial and harmful fungi}

The top 20 beneficial and harmful fungal genera with different relative abundance under 5 cropping rotation and fallow modes were analyzed by One way ANOVA. The results showed that the beneficial fungal mainly included Mortierella 、 unidentified_Mortierellales_sp. and Atractiella. The harmful fungal mainly included Alternaria, Fusarium and Plectosphaerella. Compared with WWW, the relative abundance of beneficial fungal under rotation fallow mode was significantly increased $(\mathrm{P}<0.05)$; The Atractiella in WFP was significantly higher than the other four modes, while the WPF showed a significant decrease $(P<0.05)$ (Fig. 5). For the potential harmful fungi, different rotation fallow modes had great differences, and the total relative abundance of harmful fungi in rotation fallow decreased as a whole. Compared with WWW, the relative abundances of Fusarium and Atractiella in WFP decreased significantly $(P<0.05)$, while WRF, WPF and WFR were significantly increased; The relative abundance of Alternaria is the largest in WWW(Fig. 5). Compared with WWW, crop rotation fallow can increase the relative abundance of potentially beneficial fungal in the rhizosphere and reduce harmful fungal to a certain extent to maintain soil health. 


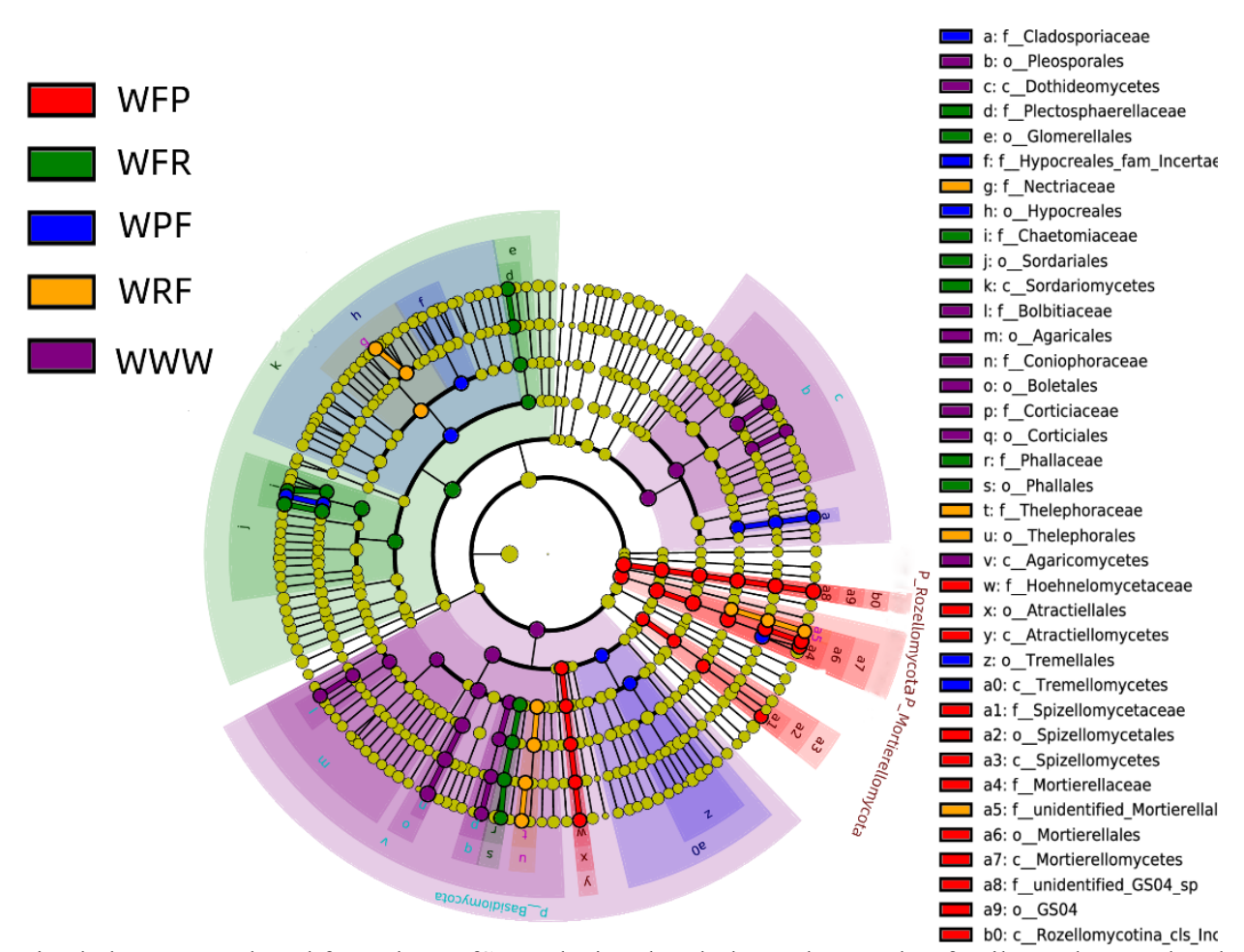

Fig. 4. Taxonomic cladogram produced from the LEfSe analysis. The phylum, class, order, family, and genus levels are listed in order from inside to outside of the cladogram, and the labels for levels of the family and genus are abbreviated using a single letter
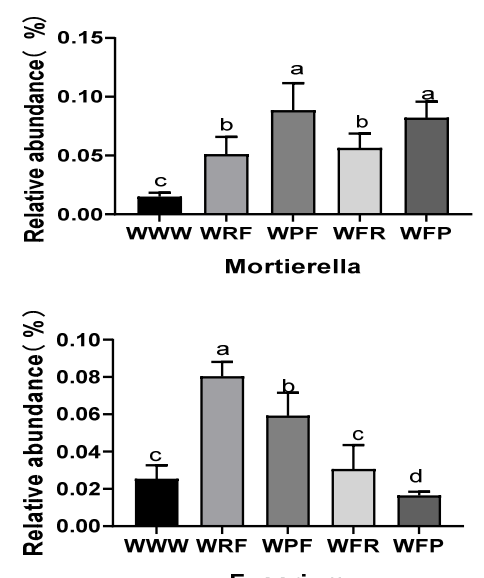

Fusarium
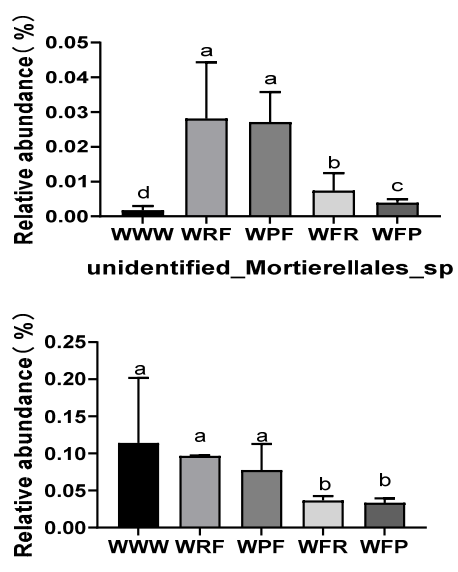

Alternaria
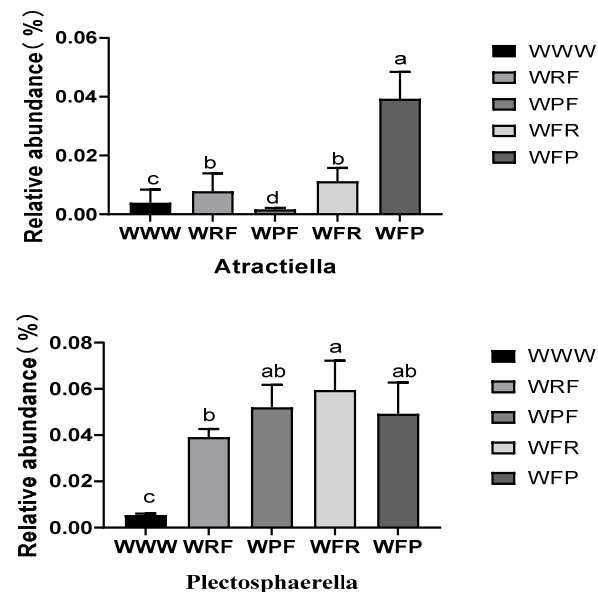

Fig. 5. Relative abundance of potentially and potentially pathogenic fungi

\section{DISCUSSION}

\subsection{Rhizosphere fungi diversity of spring wheat driven by crop rotation and crop planting sequence}

Compared with WWW, the Shannon and Simpson indices increased in different degrees, while the Chao1 and ACE indices decreased, indicating that the rotation fallow could increase the rhizosphere fungal diversity of spring wheat, and eliminate a few specific fungi. The Shannon index of WFP, WRF, WPF and WFR was significantly different, indicating that different crop species and crop planting order had different effects on rhizosphere fungal diversity, this is basically similar to the results of previous studies. Tang et al. [17] and Li et al. [18] found that the Shannon index of black pepper and Salvia miltiorrhiza decreased significantly in longterm continuous cropping, and the rhizosphere soil fungal diversity decreased significantly. Liu et al. [19] found that different crops had different responses to long-term continuous cropping fungi diversity. Yao et al. [20] found that, compared with rotation, continuous cropping significantly improved the Shannon index and ACE index of the fungal community, but no significant difference was found in the Chao index, which was inconsistent with the results of this study. The main reason is that the crop species and planting order are different. Different crops have different nutrient preferences and different root exudates during the growth and development process, thus affecting the diversity of fungal community [19]. 
PCoA results showed that rotation fallow mode was the main factor leading to the change of rhizosphere fungal community structure. Rhizosphere fungal community structure and rotation fallow mode of WWW had the most significant differences. WRF, WPF and WFR have relatively high similarity in fungal community structure, which is similar to the research results of Wang et al. [21]. Wang et al. 's study pointed out that the rhizosphere fungal community structure changed significantly in continuous cropping of fluecured tobacco, and the change became more obvious with the increase of continuous cropping years. The rhizosphere fungal community structure of soy-wheatflue-cured tobacco, soy-corn-flue-cured tobacco and corn-soy-soy-flue-cured tobacco were highly similar. The main reason is that long-term continuous cropping of crops will lead to the specific increase or decrease of some nutrients in the soil. Through crop rotation, crop residues become diversified, causing soil nutrients to develop towards diversity, driving fungal complexity and diversity to avoid large changes in them, to some extent, the community structure is similar.

\subsection{Crop rotation and crop planting order changed rhizosphere fungal species composition of spring wheat.}

In this study, we found that there were significant differences in rhizosphere fungal community composition under different rotation fallow modes. At the phylum level, Ascomycota (32.72-47.99\%), Basidiomycota (8.39-34.21\%) and Mortierellomycota $(2.05-18.42 \%)$ were the dominant fungi in the rhizosphere of spring wheat, and their total relative abundance WWW was significantly higher than that of the rotation fallow mode. Compared with WWW, the relative abundance of Mortierellomycota and Chytridiomycota increased significantly, while that of Basidiomycota decreased significantly, which was consistent with previous studies. Nan et al. [22] pointed out that soil fungal community was mainly composed of Ascomycota, Basidiomycota and Mortierellomycota. Yuan et al. [23] believe that Mortierellomycota can be used to indicate whether the soil is healthy, and the relative abundance of Mortierellomycota in healthy soil is relatively high. The relative abundance of the rotated fallow Mortierellomycota increased significantly, indicating that the rotated fallow soil was relatively healthy. Basidiomycota can be roughly divided into saprophytic fungi, symbiotic fungi and pathogenic or parasitic fungi. The relative abundance of Basidiomycota is related to crop species and soil types. Liu et al. [24] et al. found that Basidiomycota and relative abundance decreased significantly after 3 years of continuous soybean cropping compared with soybean and maize rotation, which was inconsistent with the results of this study. The reason may be the variety of crop plants, farming methods and so on.

Another interesting finding in this study was that the relative abundance of Rozellormycota in WFP was significantly higher than that in the other four modes
$(\mathrm{P}<0.05)$. Because Rozellormycota lacks a cell wall, it can obtain nutrients through phagocytosis, unlike other fungi, which can only absorb nutrients through osmosis. Such fungal decompose organic matter extracellular by secreting enzymes, and then absorb nutrients through diffusion [25]. The cell wall not only maintains its structure but also helps it resist external hazards. The significant increase in the relative abundance of Rozellormycota in WFP also indicates that the soil conditions under this model are relatively mild and can meet the growth and reproduction requirements.

At the genus level, we found that there were significant differences in the effects of different rotation fallow modes on genera. The WWW mode significantly increased the relative abundance of Coniophora and Chaetomiaecum-Unidentified. However, the relative abundance of Mortierella, Plectosphaerella and Mortierellaceae-Unidentified was significantly decreased $(\mathrm{P}<0.05)$. The relative abundance of Alternaria in WFP was the lowest, significantly lower than that of WWW, WRF and WPF $(\mathrm{P}<0.05)$. Coniophora and Chaetomiaceae belong to Basidiomycota and Ascomycota, respectively. Most of them can degrade cellulose and lignin [26-27]. The abundant residue of straw in WWW is conducive to the growth and reproduction of Coniophora and Chaetomiaceae, which also increases the relative abundance of Basidiomycota and Ascomycota in WWW to a certain extent. Mortierella and Mortierellaceae-Unidentified belong to Mortierellomycota. The relative abundance of Mortierellomycota was significantly increased in the rotation fallow modes. This resulted in a significant increase in the relative abundance of Mortierella and Mortierellaceae-unidentified. Some studies have pointed out that continuous cropping of some plants can increase the relative abundance of Mortierella, Alternaria and Fusarium, which has some similarities and differences with this study. This is mainly because there are many factors that influence changes in microbial community composition. For example, changes in soil physical and chemical properties, host plant types, crop sequences and microbial interactions [28-29].

\subsection{Optimizing the abundance of potentially beneficial and harmful fungi by rotation fallow}

The results showed that compared with WWW, rotation fallow could increase the relative abundance of potential beneficial fungal in the rhizosphere of spring wheat, and reduce the relative abundance of harmful fungal to a certain extent to maintain soil health. Compared with WWW, the rotation fallow mode significantly increased the relative abundance of beneficial fungi of Mortierella, Mortierellaceae-unidentified sp. and Atractiella $(P<0.05)$. Mortierella is an antagonistic plant pathogenic fungus that plays a certain role in the prevention and control of plant diseases [30-31]. Atractiella is a kind of plant growth promoting fungal, which is widely distributed in crop roots and can significantly promote seedling growth and photosynthetic rate [32]. Liu et al. [19] pointed out that relative abundance of Mortierella significantly 
increased under wheat-corn-soybean rotation mode compared with wheat continuous cropping and maize continuous cropping. Liu et al. [19] pointed out that the relative abundance of Mortierella under wheat-cornsoybean rotation mode was significantly increased compared with that under wheat continuous cropping and maize continuous cropping, but significantly lower than that under long-term continuous cropping of soybean, which was similar and different from the results of this study. The main reason for the difference is that the research crops are different. Crops prefer beneficial microorganisms through root exudates to help them resist biotic and abiotic stresses [3].

For the potential harmful fungi, there were great differences among different rotation fallow modes, and the total relative abundance of harmful fungi decreased in general. Compared with WWW, the relative abundance of Fusarium and Alternaria in WFP decreased significantly, while that of WRF, WPF and WFR increased significantly. The relative abundance of Alternaria is the highest in WWW. This study is similar to previous studies. Bai et al[33] and Liu et al.[19] showed that long-term continuous cropping of soybean would lead to a significant decrease in the relative abundance of Fusarium and Alternaria. Wei et al. [34] found that Fusarium SPP., a pathogen of soybean in soil after 20 years of continuous cropping. The population density was significantly lower than that of soybean rotation, which was inconsistent with the results of this study. It may be caused by different kinds of crops. In addition, Wei et al. Used the traditional culture method to analyze the relative abundance of Fusarium, while this paper used the high-throughput sequencing technology to study the species abundance. Different methods may also cause bias in the results. Fusarium and Alternaria are widely distributed in nature and contain a variety of plant pathogens, which can cause diseases of a variety of crops, such as wheat scabblight, cotton Fusarium wilt and soybean root rot [19]. Plectosphaerella can effectively use a variety of carbon and nitrogen sources, and can infect eggplant, pepper, rape and other crops [35]. In this study, the relative abundance of Plectosphaerella decreased significantly in WWW. This may be related to crop species and the infection characteristics of Plectosphaerella. The rotation crops in this study include potato and rape of the same family as eggplant and pepper, which may be easier to infect and colonize than wheat, a grass crop.

\section{Conclusion}

Compared with WWW, Shannon and Simpson index increased, while Chaol and ACE index decreased. The results showed that rotation and fallow could increase the diversity of rhizosphere fungi of spring wheat, and eliminate a small number of specific fungi. The effects of crop types and planting order on the diversity of rhizosphere fungi were different. At the phylum level, the top three fungi in the rhizosphere of spring wheat were Ascomycota (32.72-47.99\%), Basidiomycota (8.39$34.21 \%)$ and Mortierellomycota (2.05-18.42\%).
Compared with WWW, the relative abundance of Mortierellomycota and Chytridiomycota increased significantly, but the relative abundance of Basidiomycota decreased significantly. At the genus level, compared with WWW, crop rotation and fallow could increase the relative abundance of potentially beneficial fungi in the rhizosphere, and reduce the relative abundance of harmful fungi to a certain extent, thus maintaining soil health. Compared with WWW, the rotation fallow mode significantly increased the relative abundance of beneficial fungi of Mortierella, Mortierellaceae-unidentified sp. and Atractiella $(\mathrm{P}<0.05)$. Compared with WWW, the total relative abundances of the harmful fungi Fusarium, Plectosphaerella and Alternaria decreased in the rotation fallow mode. The Atractiella in WFP was significantly higher than that in the other four modes, but the relative abundance of Fusarium and Alternaria were significantly decreased $(\mathrm{P}<0.05)$. In general, cropping rotation and fallow mode can increase rhizosphere fungal diversity, increase beneficial species and abundance, and affect rhizosphere fungi community composition of spring wheat. In addition, WFP can lead to the development of rhizosphere fungal community structure in favor of plant health.

\section{Acknowledgments}

This work was supported by the National Natural Science Foundation of China (31860356), the National Key Research and Development Program of China (2019YFC0507600, 2019YFC0507605); The Major Project of Science and Technology in Inner Mongolia of China(2019ZD009, zdzx2018017) and the Leading Talent Project of "Grassland Talents" in Inner Mongolia of China.

\section{References}

1. Latef A, Hashem A, Rasool S, J, Arbuscular mycorrhizal symbiosis and abiotic stress in plants: A review, PLant Biol, 59, 407-426 (2016).

2. S Jiao, Z H Peng, J J Qi, et al, Linking BacterialFungal Relationships to Microbial Diversity and Soil Nutrient Cycling, mSystems, 6, e01052-20 (2021).

3. M J Kwak, H G Kong, Rhizosphere microbiome structure alters to enable wilt resistance in tomato, Nat Biotechnol, 36, 4 1-10 (2018).

4. Coats VC, Rumpho ME, The rhizosphere microbiota of plant invaders: an overview of recent advances in the microbiomics of invasive plants, Front. Microbiol., 5, 368 (2014).

5. H Setälä, Mclean M A, Decomposition rate of organic substrates in relation to the species diversity of soil saprophytic fungi, Oecologia, 139, 1, 98-107 (2004).

6. Jin H U, Meng D L, Liu X D, et al, Response of soil fungal community to long-term chromium 
contamination,Trans. Nonferrous Met. Soc. China, 28, 9, 1838-1846 (2018).

7. Ashworth, A.J.J., DeBruyn, J.M.M., Allen, F.L.L., Radosevich, M., Owens, P.R.R., Microbial community structure is affected by cropping sequences and poultry litterunder long-term notillage, Soil Biol. Biochem., 114, 210-219 (2017).

8. Venter Z S, Jacobs K, Hawkins H J, The impact of crop rotation on soil microbial diversity: A metaanalysis, Pedobiologia, 215-223 (2016).

9. W G Jie, B Li, W J Yu, B Y Cai, Analysis of interspecific relationships between Funneliformis mosseae and Fusarium oxysporum in the continuous cropping of soybean rhizosphere soil during the branching period, BIOCONTROL SCI TECHN, 25, 9, 1036-1051(2015).

10. X L Tan, Study on soil microbial diversity, enzyme activity and root exudates of potato under rotation mode, Gansu Agricultural University, (2016).

11. Adams R I,Miletto M,Taylor J W, et al, Dispersal in miscrobes:fungi in indoor air are doiminated by outdoor air and show dispersal limitation at short distances, ISME J, 7, 7, 1173-1262 (2013).

12. L L Nan, J H Tan, Q E Guo, Effects of rotation fallow on soil fungi in semiarid area of Loess Plateau, Acta ecologica Sinica, 20, 23, 8582-8592 (2020).

13. R C Edgar, UPARSE: highly accurate OTU sequences from microbial amplicon reads, Nature Methods, Nat Methods, 10, 10, 996-998 (2013).

14. Caporaso J G, Kuczynski J, Stombaugh J, QIIME allows analysis of high-throughput community sequencing data, Nature Methods,7 , 5, 335-336 (2010).

15. Q Wang, G M Garrity, J M Tiedje, J R Cole, Naive Bayesian classifier for rapid assignment of rRNA sequences into the new bacterial taxonomy, Appl. Environ. Microbiol., 73, 16, 5261-5267 (2007).

16. P D Schloss, Gevers D, Westcott S L, Reducing the effects of PCR amplification and sequencing artifacts on 16S rRNA-based studies, PLoS One,6 , 12, e27310 (2011).

17. Tang J, Xue Z, Daroch M, Ma J, Ann Microbiol NLM, Impact of continuous Salvia miltiorrhiza cropping on rhizosphere actinomycetes and fungi communities, 65, 1267-1275 (2015).

18. Z G Li, C Zu, C Wang, J F Yang, $\mathrm{H} \mathrm{Yu,} \mathrm{H} \mathrm{S} \mathrm{Wu,}$ Different responses of rhizosphere and nonrhizosphere soil microbial communities to consecutive Piper nigrum L. monoculture, Sci. Rep., 6, 1-8 (2016).

19. Liu H, Pan F J, Han X Z, et al, A comprehensive analysis of the response of the fungal community structure to long-term continuous cropping in three typical upland crops, J INTEGR AGR, 19, 3, 866880 (2020).
20. Y H Yao, Y L Wang, X H Yao, L K An, Y X Bai, X $\mathrm{Li}, \mathrm{K} \mathrm{L} \mathrm{Wu}$, Effects of different planting patterns on microbial community structure in rhizosphere soil of highland barley, Journal of NWSTUAF, 4, 1-10 (2 021).

21. G H Wang, G W Zhao, H W Zhao, et al, Changes of microbial community structure in rhizosphere soil of Flue-cured Tobacco under different planting systems, Heilongjiang Agricultural Sciences, 11, 26-30 (2011).

22. L L Nan, J HTan, Q N Guo, Effects of rotation fallow on soil fungi in semiarid area of Loess Plateau, Acta ecologica Sinica, 40, 23, 8582-8592 (2020).

23. J Yuan, $\mathrm{T}$ Wen, $\mathrm{H}$ Zhang, et al, Predicting disease occurrence with high accuracy based on soil macroecological patterns of Fusarium wilt, ISME J, (2020).

24. Z X Liu, J J Liu, Z H Yu, et al, Long-term continuous cropping of soybean is comparable to crop rotation in mediating microbial abundance, diversity and community composition, Soil Tillage Res, 197(2020).

25. M Turner, The evolutionary tree of fungi grows a new branch, Nature News, 11 May 2011 [2014-1031]. doi:10.1038/news.2011.285.

26. H Kauserud, Svegården IB, C Decock, et al, Hybridization among cryptic species of the cellar fungus Coniophora puteana (Basidiomycota), Mol Ecol, 16, 2, 389-399 (2010).

27. Y Z Guo, Phylogeny and taxonomic identification of chaetomiaceae fungi, Northwest University of agriculture and forestry science and technology, 2012.

28. C. H.Kong, P. Wang, H Zhao, et al, Impact of allelochemical exuded from allelopathic rice on soil microbial community, Soil Biol. Biochem., 40, 1862-1869 (2008).

29. A. J. Ashworth, De Bruyn, et al, Microbial community structure is affected by cropping sequences and poultry litter under long-term notillage, Soil Biol. Biochem., 114, 210-219 (2017).

30. F Pan., A. G. Xue, N. B.McLaughlin, et al, Colonization of Clonostachys rosea on soybean root grown in media inoculated with Fusarium graminearum, Plant Soil, 63, 564-569 (2013).

31. Z.Shen, C. R.Penton, C.Xue, et al, Banana fusarium wilt disease incidence is influenced by shifts of soil microbial communities under different monoculture spans, Microb Ecol, 75, 739-750 (2018).

32. Z L Yuan, X Y Pan, J Wei, et al, Symbiotic system of forest trees and its mechanism of action: a case study of poplar, Acta Zoologica Sinica, 39, 1, 385401(2019).

33. L Bai, J Q Cui, W G Jie, et al, Analysis of the community compositions of rhizosphere fungi in soybeans continuous cropping fields, MICROBIOL RES, 180, 49-56 (2015). 
34. W Wei, Y Xu, S Li, et al, Developing suppressive soil for root diseases of soybean withcontinuous long-term cropping of soybean in black soil of Northeast China, Acta Agriculturae Scandinavica, Section B - Soil \& Plant Science., 65, 279-285 (2015).
35. X U Jing, De X U, L J Wang, et al, Biological Characteristics on Plectosphaerella cucumerina of Plectosphaerella Tomato Wilt Causal Agent Journal of Shenyang Agricultural University, (2014). 